\title{
Correction to: A novel generation 1928zT2 CAR T cells induce remission in extramedullary relapse of acute lymphoblastic leukemia
}

Jianyu Weng ${ }^{1 \dagger}$, Peilong Lai ${ }^{1 \dagger}$, Le Qin ${ }^{2,3+}$, Yunxin Lai ${ }^{2,3}$, Zhiwu Jiang ${ }^{2,3}$, Chenwei Luo ${ }^{1}$, Xin Huang ${ }^{1}$, Suijing Wu', Dan Shao ${ }^{4}$, Chengxin Deng ', Lisi Huang', Zesheng Lu', Maohua Zhou', Lingji Zeng', Dongmei Chen ${ }^{2,3}$, Yulian Wang ${ }^{1}$, Xiaomei Chen', Suxia Geng ${ }^{1}$, Robert Weinkove ${ }^{5}$, Zhaoyang Tang ${ }^{6,7}$, Chang He ${ }^{8}$, Peng Li ${ }^{2,3 *}$ and Xin Du ${ }^{1 *}$

\section{Correction to: J Hematol Oncol https://doi.org/10.1186/s13045-018-0572-x}

The original article [1] contains an error in authorship whereby author, Robert Weinkove's name is mistakenly inverted. The configuration noted in this Correction article should be considered instead along with author's updated affiliation.

\section{Author details}

'Department of Hematology, Guangdong General Hospital, Guangdong Academy of Medical Sciences, Guangzhou 510080, China. ${ }^{2}$ Key Laboratory of Regenerative Biology, South China Institute for Stem Cell Biology and Regenerative Medicine, Guangzhou Institutes of Biomedicine and Health, Chinese Academy of Sciences, Guangzhou 510530, China. ${ }^{3}$ Guangdong Provincial Key Laboratory of Stem Cell and Regenerative Medicine, South China Institute for Stem Cell Biology and Regenerative Medicine, Guangzhou Institutes of Biomedicine and Health, Chinese Academy of Sciences, Guangzhou 510530, China. ${ }^{4}$ Department of PET Center, Guangdong General Hospital and Guangdong Academy of Medical Sciences, Guangzhou 510080, China. ${ }^{5}$ The Malaghan Institute of Medical Research, Wellington, New Zealand. ${ }^{6}$ Guangdong Zhaotai InVivo Biomedicine Co. Ltd., Guangzhou

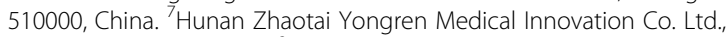
Changsha 410000, China. ${ }^{8}$ State Key Laboratory of Ophthalmology, Zhongshan Ophthalmic Center, Sun Yat-Sen University, Guangzhou 510060, People's Republic of China.

Published online: 20 November 2019

\section{Reference}

1. Weng J, Lai P, Qin L, Lai Y, Jiang Z, Luo C, et al. A novel generation 1928zT2 CAR T cells induce remission in extramedullary relapse of acute lymphoblastic leukemia. J Hematol Oncol. 2018;11:25 https://doi.org/10. 1186/s13045-018-0572-x.

\footnotetext{
*Correspondence: li_peng@gibh.ac.cn; miyadu@hotmail.com

The original article can be found online at https://doi.org/10.1186/s13045018-0572-x

${ }^{\dagger}$ Jianyu Weng, Peilong Lai and Le Qin contributed equally to this work.

${ }^{2}$ Key Laboratory of Regenerative Biology, South China Institute for Stem Cell Biology and Regenerative Medicine, Guangzhou Institutes of Biomedicine and Health, Chinese Academy of Sciences, Guangzhou 510530, China 'Department of Hematology, Guangdong General Hospital, Guangdong Academy of Medical Sciences, Guangzhou 510080, China

Full list of author information is available at the end of the article
}

(c) The Author(s). 2019 Open Access This article is distributed under the terms of the Creative Commons Attribution 4.0 International License (http://creativecommons.org/licenses/by/4.0/), which permits unrestricted use, distribution, and reproduction in any medium, provided you give appropriate credit to the original author(s) and the source, provide a link to the Creative Commons license, and indicate if changes were made. The Creative Commons Public Domain Dedication waiver (http://creativecommons.org/publicdomain/zero/1.0/) applies to the data made available in this article, unless otherwise stated. 